\title{
Microfluidic behaviour of perfluoropolyether fluids in poly(dimethylsiloxane) micro-channels
}

\author{
Ilenia Viola ${ }^{a}$, Giuseppe Ciccarella ${ }^{\mathrm{a}}$, Pierangelo Metrangolo ${ }^{\mathrm{b}, *}$, \\ Giuseppe Resnati ${ }^{\mathrm{b}, * *}$, Roberto Cingolani ${ }^{\mathrm{a}}$, Giuseppe Gigli ${ }^{\mathrm{a}, * * *}$ \\ ${ }^{a}$ National Nanotechnology Laboratory (NNL) of CNR, INFM, Distretto tecnologico ISUFI and Dipartimento di Ingegneria dell'Innovazione, \\ Università degli Studi di Lecce, via Arnesano, I-73100 Lecce, Italy \\ ${ }^{\mathrm{b}}$ Nanostructured Fluorinated Materials Laboratory (NFMLab), Dipartimento di Chimica, Materiali e Ingegneria Chimica "G. Natta", \\ Politecnico di Milano, via Mancinelli 7, I-20131 Milano, Italy
}

Received 13 July 2007; received in revised form 6 August 2007; accepted 6 August 2007

Available online 15 August 2007

Dedicated to Prof. Kenji Uneyama on the occasion of his receiving the 2007 ACS Award for Creative Work in Fluorine Chemistry.

\begin{abstract}
Two different perfluoropolyether-based fluids, namely the unfunctionalized GALDEN SV90 ${ }^{\circledR}$ and the dihydroxy derivative FOMBLIN ZDOL $^{\mathbb{R}} 2000$ were employed as liquid samples in a poly(dimethylsiloxane) (PDMS) microfluidic setup, fabricated by soft-lithography techniques. The results of our investigation were compared with the behaviour of the low viscosity and high-fragility polyurethane structural adhesive $\left(\right.$ NOA $\left.72{ }^{\mathbb{R}}\right)$, that is well known as an excellent material for the fabrication of sub-micrometer structures by soft-lithography techniques, and whose structural elastic properties inside restricted geometric systems have been recently investigated.
\end{abstract}

(C) 2007 Elsevier B.V. All rights reserved.

Keywords: Perfluoropolyethers; Microfluidics; Surface effects

\section{Introduction}

Much effort has been directed to the study of the properties of solid-liquid interface during the displacement of liquids inside geometrically restricted structures. A fine control of the dynamics in a microfluidic setup seems to be a prevalent tool to effectively address manifold operations inside integrated fluidic circuits (i.e. lab-on-chips, LOCs). Because of the profound influence that the fluorine-containing liquids can have upon the physical and chemical properties at the interface [1], we have employed two different perfluoropolyether-based fluids, avail-

\footnotetext{
*Corresponding author. Tel.: +39 0223993041; fax: +390223993180.

E-mail address: pierangelo.metrangolo@polimi.it (P. Metrangolo) URL: http://nfmlab.chem.polimi.it

**Corresponding author. Tel.: +39 0223993032; fax: +39 0223993180 . E-mail addresses: giuseppe.resnati@polimi.it (G. Resnati) URL: http://nfmlab.chem.polimi.it

*** Corresponding author. Tel.: +39 0832 298216; fax: +390832 298238 . E-mail addresses: giuseppe.resnati@polimi.it (G. Resnati) $U R L:$ http://nfmlab.chem.polimi.it
}

able under the trade name GALDEN SV90 ${ }^{\circledR}$ and FOMBLIN Z-DOL ${ }^{\circledR} 2000$ (Solvay-Solexis), as liquid-samples inside a poly(dimethylsiloxane) (PDMS) micro-channels structure.

GALDEN $\quad \mathrm{SV} 90^{\circledR} \quad\left(\mathrm{CF}_{3}-\left[\left(\mathrm{OCF}_{2} \mathrm{CF}_{2} \mathrm{CF}_{2}\right)_{m}-\left(\mathrm{OCF}_{2}\right)_{n}-\right]\right.$ $\mathrm{OCF}_{3}$ ) is mainly employed for its thermal resistance, nontoxicity, excellent dielectric properties, no flash or fire point and low viscosity. Typical applications include heat transfer, electronic reliability testing and coatings [2]. FOMBLIN Z-DOL $2000^{\mathbb{R}}\left(\mathrm{HOCH}_{2} \mathrm{CF}_{2} \mathrm{O}-\left(\mathrm{CF}_{2} \mathrm{CF}_{2} \mathrm{O}\right)_{p}-\left(\mathrm{CF}_{2} \mathrm{O}\right)_{q}-\mathrm{CF}_{2} \mathrm{CH}_{2} \mathrm{OH}\right)$ is a high performance lubricant with applications in the chemical, electronic, nuclear and data processing industries [3]. Details of the material properties are reported in Table 1.

In the last years, PDMS surface chemistry has been gaining increasing attention because of its potential applications especially in the diagnostic industry, where PDMS is the main component for the realization of micron and nanometre structures over a large area inside functional integrated devices [4,5]. In general, the investigation of the fluiddynamics properties in micro and nano-channels allows the in situ control of the liquid flow, which is required for the fabrication of microfluidics device components. A total 
Table 1

Material properties at room temperature

\begin{tabular}{|c|c|c|c|c|c|c|c|}
\hline & $\begin{array}{l}\text { Molecular } \\
\text { weight (amu) }\end{array}$ & $\begin{array}{l}\text { Viscosity } \\
\text { (cSt) }\end{array}$ & $\begin{array}{l}\text { Boiling } \\
\text { point }\left({ }^{\circ} \mathrm{C}\right)\end{array}$ & $\begin{array}{l}\text { Dielectric } \\
\text { strength }(\mathrm{kV})\end{array}$ & $\begin{array}{l}\text { Density } \\
\left(\mathrm{g} / \mathrm{cm}^{3}\right)\end{array}$ & $\begin{array}{l}\text { Surface tension } \\
(\mathrm{dyn} / \mathrm{cm})\end{array}$ & $\begin{array}{l}\text { Polydispersity } \\
\text { (Mw/Mn) }\end{array}$ \\
\hline Galden SV90 & 460 & 0.75 & 92 & 34 & 1.69 & 16 & \\
\hline Fomblin Z-DOL 2000 & 2000 & 85 & & & 1.81 & 24 & 1.5 \\
\hline NOA72 & $<1000$ & 0.16 & & 456 & & 38 & \\
\hline
\end{tabular}

integration of the peripheral functions onto single microchips can be possible only when a great variety of chemical and biological processes can be implemented, including technologies for novel microfluidic components integrating more than one function. These functions could be filtering, preconcentration, separation, sorting, mixing, reactants confinement, local chemistry and flow control. The role of the mentioned fluoropolymers in the development of such components can be of interest especially for miniaturized systems, whose typical dimensions are at sub-millimetre scale and thus are characterized by high values of surface-tovolume ratio $(S / V)$. In such geometrically confined systems, also well known as high $S / V$ systems, the driving motion is strongly influenced by surface interactions and localization phenomena, which dominate over the bulk dynamics and usually indicate a fast diffusion regime.

Therefore, the analysis of the dynamical properties of different liquids in confined systems is thus proposed, considering the time condition for a fast diffusion regime as $T_{\mathrm{S}} \gg T_{\mathrm{D}} . T_{\mathrm{S}}$ is the surface time, namely the average time of each molecule's interaction with the surface $T_{\mathrm{S}} \cong \rho^{-1}(V /$ $S$ ), with $\rho$ related to the molecular relaxation rate near the surface [4] and $T_{\mathrm{D}}$ is the molecular diffusion time according to the Einstein equation $T_{\mathrm{D}} \cong D^{-1}(V / S)$ [5]. In this work, we report an in situ and real-time microfluidic technique as a direct tool for the analysis of the most relevant properties of fluorine-based liquids in a confined system during the operational regime. For this purpose, we have used as fluidic confined structure a network of microchannels with a rectangular cross-sectional area (Fig. 1), obtained by the conformal contact of a PDMS microstructured mould, fabricated by a soft-lithography technique, and a flat $\mathrm{SiO}_{2} / \mathrm{Si}$ substrate.

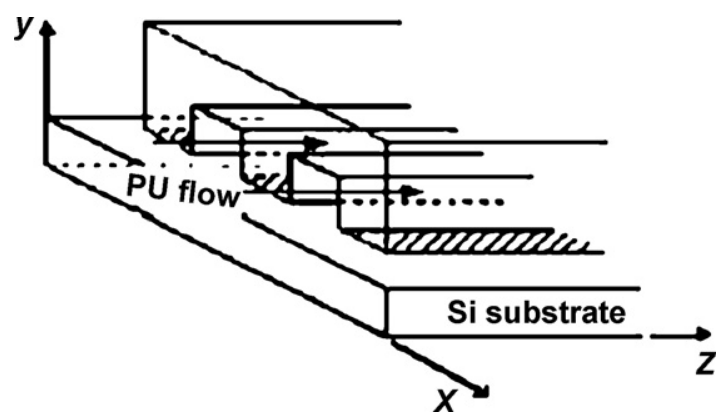

Fig. 1. Schematic of the MIMIC process (features not in scale). The conformal contact between the Si support and a PDMS mould induces the PU to flow inside the micro-channels.

\section{Results and discussion}

Our microfluidic research bases the capillary condition as the macroscopic motion of a fluid system under the influence of its own surface and interfacial forces, and results from two opposing forces: the liquid adhesion and the cohesive surface tension.

In general, in the absence of other external forces, capillary flow is controlled by the interplay of the various interfacial tensions, the geometry of the solid-liquid-vapour interface and the geometry of the solid interface. For the analysis of our microfluidic system, we choose to focus the attention on the energetic conditions, such as surface tension and contact angle, both of the liquid and the solid interface and the channels geometry. Besides, we assume to neglect the effect of the geometry at the solid-liquid-vapour interface because we have kept constant the experimental contour conditions.

In a horizontal capillary environment (Fig. 1), the fluid driving motion is generally governed by the Laplace equation for the capillary pressure drop [4-6]. Capillary forces provide an alternative means to create a pressure difference in different regions of small hydraulically connected conducts, where the direction of the resulting flow allows the pressure difference to be decreased. Recent literature has demonstrated that the Laplace equation is valid up to nanometre length scale, and that on this scale the filling of nanochannels by capillarity is dominated by the wetting properties of the channel walls [7].

Differences of the meniscus shape during the displacement of the polyurethane, NOA72, and FOMBLIN Z-DOL are well evidenced in Fig. 2a and b. In particular, the dynamic behaviour of NOA72 (Fig. 2a) is characterized by a certain hydrophilic behaviour with a contact angle of $\theta=80^{\circ}$, whereas the concave front of the fluorinated liquid (Fig. 2b) reveals a hydrophobic behaviour with a contact angle of $\theta=120^{\circ}$.

In a horizontal capillary environment (as the one sketched in Fig. 1) the fluid driving motion is generally influenced both by the interfacial mechanical tension and by the curvature of the different phases, as can be clearly expressed by the Laplace equation for the capillary pressure drop $\Delta p=\left(2 \gamma_{\mathrm{LV}} \cos \theta\right) / r$, where $\gamma_{\mathrm{LV}}$ is the free energy at the liquid-vapour interface and $\theta$ is the wetting angle between the liquid and the capillary surface. The Laplace pressure $\Delta p$ is the pressure difference in the two phases forming the interface, required to sustain the curvature resulting from the surface tension, and represents the thermodynamic conditions that favour the filling process. For a curved interface $p$ is defined as the internal front pressure minus the external front pressure $\left(\Delta p=\Delta p_{\text {int }}-\Delta p_{\text {ext }}\right)$. Therefore, evidence of significant negative pressure can be attributed to the 

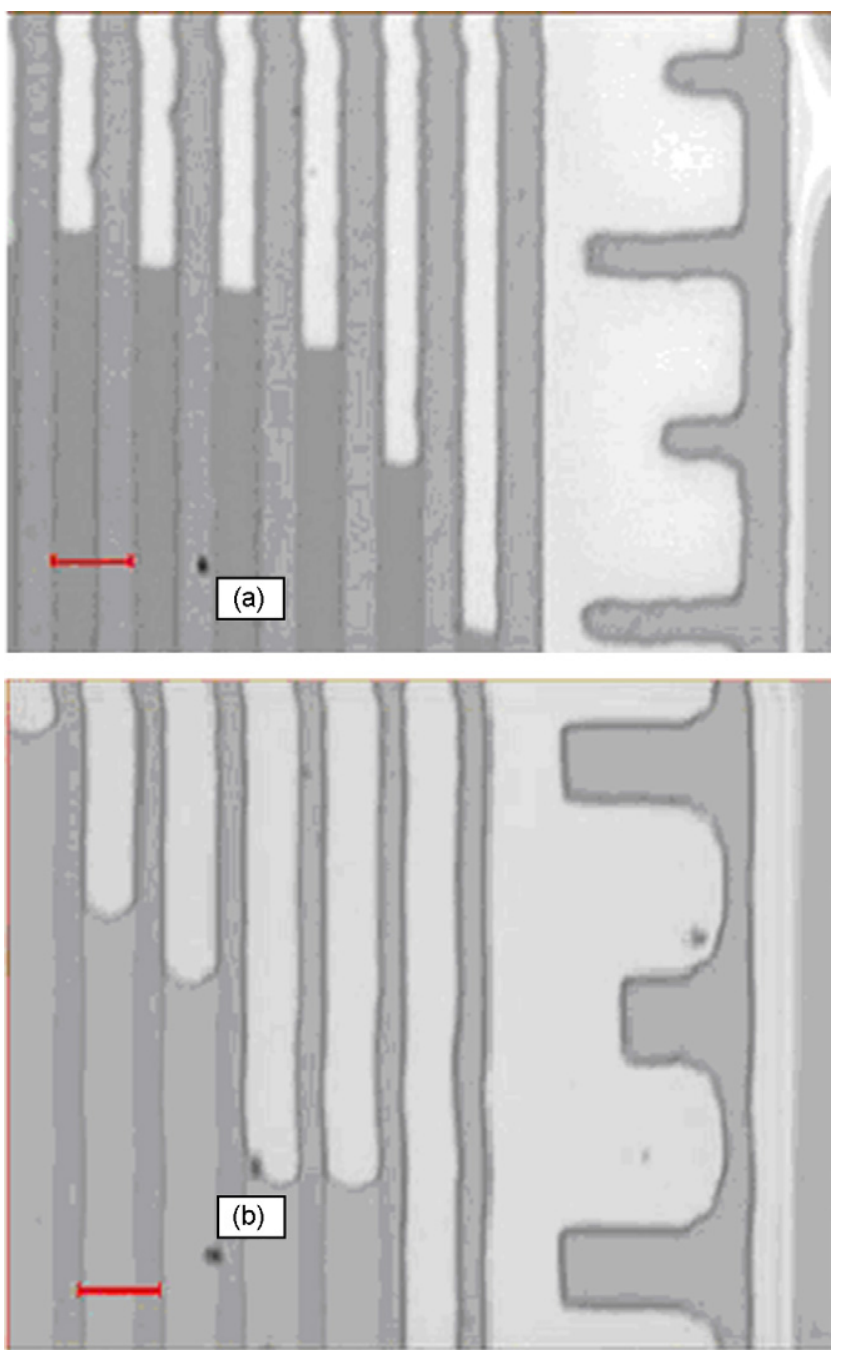

Fig. 2. MIMIC experiments evidencing the differences in meniscus shape. Picture (a) shows NOA72 ${ }^{\circledR}$. Picture (b) represents the microfluidic behaviour of FOMBLIN Z-DOL ${ }^{\circledR}$ having a more pronounced meniscus.

tensile capillary forces of the hydrophobic fluid [7]. The negative pressure of the liquid at micrometer scale induces bending of the thin channel capping layer, which results in a visible curvature of the liquid meniscus. A negative pressure can lead to evident advantages for LOC technology limiting the absorption of the liquid-sample by the PDMS walls as well as any sample leakage and allowing the confinement of polar liquid droplets containing the sample into an apolar carrier.

The balance of the wicking pressure, against the viscous drag on the wall of a circular channel (with an inner radius $r$ ), and the evolution in time of the flow rate $(Q \cong \Delta p / \eta R)$ yields the Poiseuille's Eq. (1), that relates the linear rate of liquid displacement $z$ in laminar flow to various characteristics of the fluid and capillary system $[8,9]$

$\frac{\mathrm{d} z}{\mathrm{~d} t}=r^{2} \frac{\Delta p}{8 \eta z}$

The integration of Eq. (1) represents the power-law diffusion dynamics, known as Washburn-type power-law, wherein the

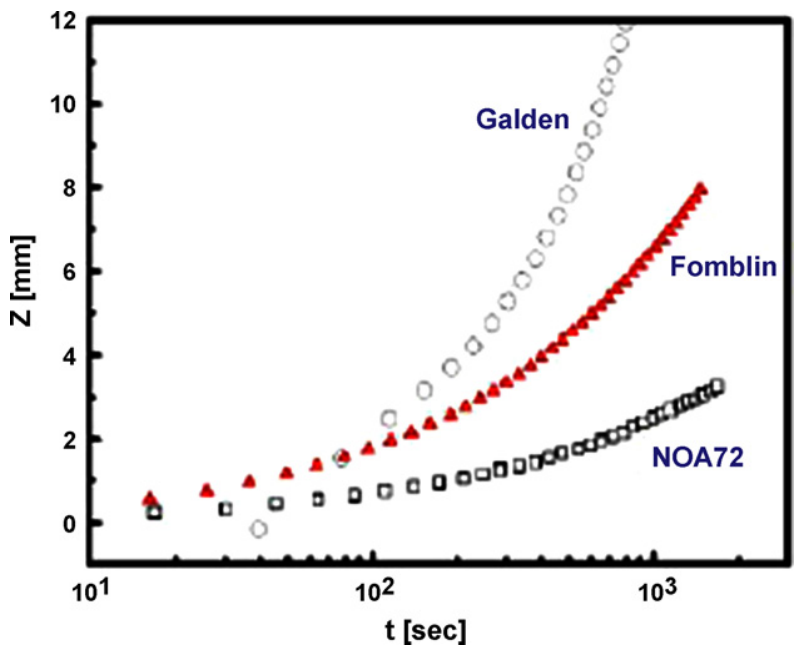

Fig. 3. Data curves of the displacement coordinate $(z)$ vs. time for the three fluids.

liquid front $z$ advances as the root square of the time [10]

$z-z_{0}=\left(\frac{r \gamma_{\mathrm{LV}} \cos \theta}{2 \eta}\right)^{1 / 2}\left(t-t_{0}\right)^{1 / 2}$

The investigation on the imbibition properties of a liquid as a function of the surface wetting and the viscoelastic behaviour was, thus, performed.

Fig. 3 shows the room temperature data curves of the displacement coordinate $(z)$ versus time $(t)$ for the three solvents. The filling velocity increases with the decrease of the surface tension of the tested liquids. Furthermore, the hydrophobic nature of the PDMS, together with the filling process of the microstructure in a dynamically confined regime, plays a determinant role in the differentiation of the microfluidic behaviour.

More importantly, investigating the effects of the elastic properties of the liquid used on the displacement dynamics is strategically turned to the fact that the use of solutions of flexible high-molecular-weight polymer inside microchannels allows an efficient mixing also at low Reynolds numbers $(R e)$. In fact, it is generally known that the presence of the polymers leads to elastic instability and elastic stresses that appear during the flow of solution of long-chain polymer, behaving as viscoelastic fluid due to its non-linear mechanical properties [11].

In fact, the elastic effects, typically present in polymer flow, grow non-linearly with the flow rate $Q$ and this can lead to strong changes in the characteristics of flow even at low velocity and high viscosity. These effects could finally allow the mixing of liquids in restricted submillimetre geometries, that is one of the main open problems for example of microfluidic systems, characterized by a laminar flow and thus by small Reynolds numbers. The reason why small amounts of polymer can significantly modify properties of the fluid is the flexibility of polymer molecules, that give non-linear mechanical properties to the elastic polymer solution, thus producing a sort of elastic turbulence that occurs at low $R e$ and realizes 
Table 2

The structural rheological parameters of the Fomblin-like fluid, obtained by the direct, in situ microfluidic approach and compared with the polyurethane properties

\begin{tabular}{lll}
\hline & Fragility coefficient $(D)$ & Temperature, $T_{0}(\mathrm{~K})$ \\
\hline NOA & 1.9 & 242.6 \\
Fomblin & 0.34 & 262.2
\end{tabular}

efficient liquids mixing. Moreover the structural parameters of a viscoelastic liquid are strongly influenced by the surface chemistry, working pressure and device fabrication process. Typical non-Newtonian fluids have, in fact, shear-rate dependent viscosity usually well-described by the VogelFulcher-Tamman (VFT) equation that expresses the temperature-dependence of viscosity $[12,13]$

$\eta(T)=\eta_{0} \exp \left[\frac{D T_{0}}{T-T_{0}}\right]$

This typical behaviour of liquid viscosity is introduced into the Washburn capillary law (Eq. (2)) in order to investigate the structural and elastic properties of a FOMBLIN-like liquid, under the real operation conditions of a microfluidic-integrated process.

Therefore, the direct, in situ, investigation was referred to the dynamic Eq. (2) of liquid displacement in the micro- and nano-meter environment, performed at different temperatures and by introducing the VFT model for the relaxation processes. The construction of an analytical ratio function was described in a previous work [4]. The ratio of the liquid displacement $z(t$, $T$ ) at two different temperatures gave the structural parameters, e.g. viscosity, fragility, and glass transition temperature, of the Fomblin-like sample filling the micro-channels network. The results reported in Table 2, showed a fragility coefficient D of FOMBLIN Z-DOL 2000 smaller than the one of NOA72.

\section{Conclusions}

The results reveal a particularly fragile behaviour of the fluorinated fluid that is related to a more dramatic decrease of the characteristic time-scale during the molecular diffusion. A more noticeable relaxation results when temperature is increased. In addition, it was shown recently that the relaxation time of the polymer chain increases with an increase of the pressure [14]. Therefore, this means that according to the VFT model, on increasing the pressure the Vogel temperature $T_{0}$ increases and the fragility $D$ decreases (see Fig. 4).

Thus the structural parameters of a polymeric solution strongly depend on the typical internal pressure of the device and we know from the Laplace equation that a capillary pressure in a microfluidic array is typically very high.

Therefore, by considering the strategic role that a fluorinebased polymer could play in microfluidics, the results show why there is the need to provide a calculation in situ of the dynamical properties of the polymer used. In fact, the flow pattern and the elastic polymer structural parameters strongly change at the micrometer scale and they are affected by the
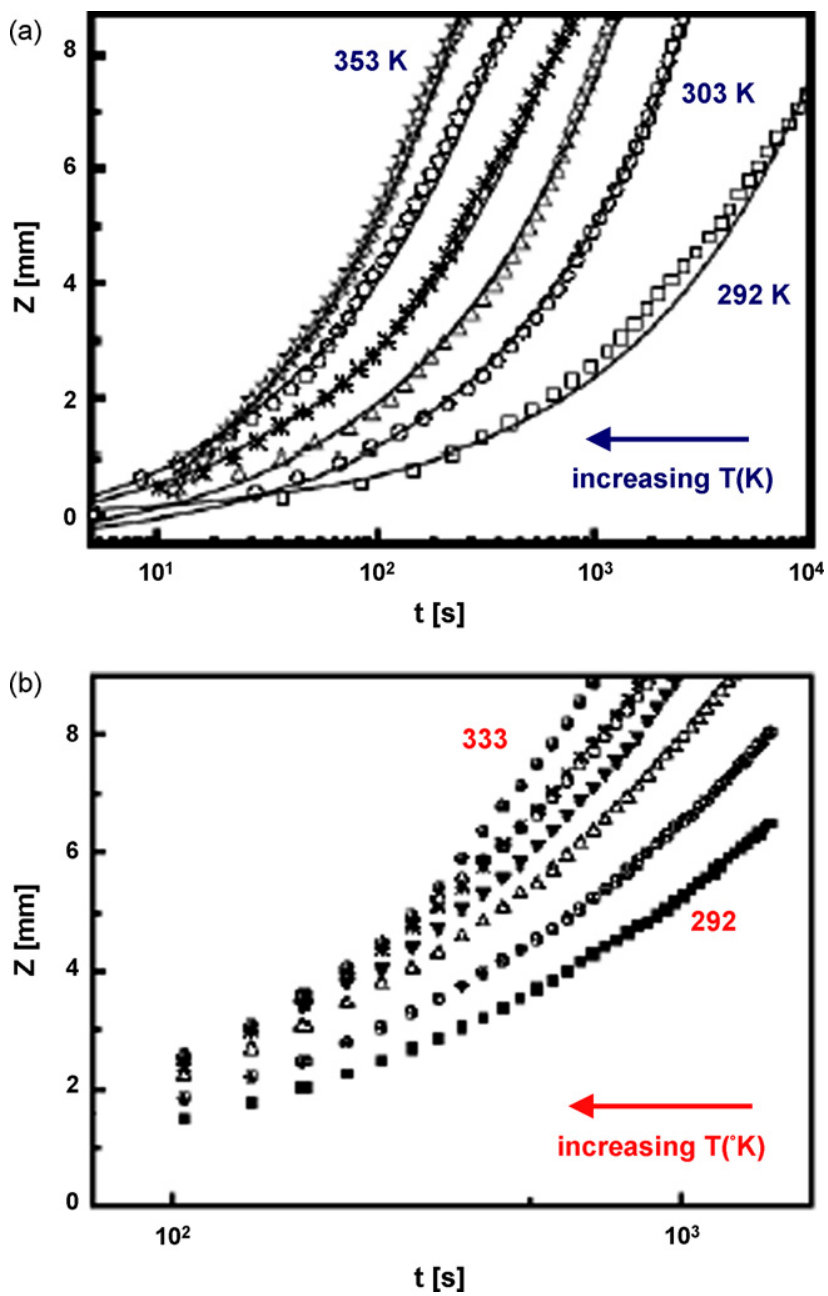

Fig. 4. Velocity of liquid displacement for FOMBLIN (a) and NOA72 (b).

energetic contributes at the solid interface, usually depending from the particular fabrication and experimental conditions.

The need of a direct analysis is confirmed by the intrinsic problems connected to the integration of functional systems in which the exact control of the working conditions is crucial for the correct operation of every component.

The behaviour of a neutral Z-Fomblin 2000 in the same microfluidic setup is under current investigation in order to study if there exists a dependence of the $z$ value on polarity or on Van der Waals type interactions in the fluid or between the fluid and the walls of the micro-channels.

\section{Experimental}

Micro-channels with a rectangular cross-sectional area (Fig. 1) were fabricated by putting in conformal contact a PDMS micro-structured mould (parallel channels, $15 \mu \mathrm{m}$ width and $1.6 \mu \mathrm{m}$ depth) with a flat $\mathrm{SiO}_{2} / \mathrm{Si}$ substrate. One side of the mould was cut perpendicularly to the channels to allow the fluid imbibition. A $10 \mu \mathrm{l}$ drop of the polymer fluid was placed at the open side, thus filling the channels by capillarity. The spontaneous capillary driving flow has been followed by an optical microscope by integrating a ruler in the master structure. 
Details about master fabrication, realization of the elastomeric replica and micro molding in capillaries (MIMIC) procedure are reported elsewhere [6,7].

The temperature of the system has been varied in the range of $292<T(\mathrm{~K})<353$ by using a hot plate in the bottom side of the $\mathrm{Si}$ substrate.

\section{References}

[1] M.K. Erhardt, R.G. Nuzzo, J. Phys. Chem. B 105 (2001) 8776-8784; D. Sianesi, G. Marchionni, R. De Pasquale, in: R.E. Banks, B.E. Smart, J.C. Tatlow (Eds.), Organofluorine Chemistry, Plenum Press, New York, 1994.

[2] H. Yamashita, E.O. Forster, M. Pompili, IEEE Trans. Electr. Insul. 28 (1993) 324-329;

P. Christian, S.M. Howdle, J.D. Irvine, Macromolecules 33 (2000) 237239;

F. Laffineur, Z. Mekhalif, L. Tristanib, J. Delhalle, J. Mater. Chem. 15 (2005) 5054-5062.

[3] K.C. Eapena, S.T. Pattona, J.S. Zabinskib, Tribology Lett. 12 (2002) 3541

J.V. Wasem, B.L. LaMarche, S.C. Langford, J.T. Dickinson, J. Appl. Phys. 93 (2003) 2202-2207;
C.M. Mate, M.F. Toney, K.A. Leach, IEEE Trans. Magnet. 37 (2001) $1821-1823$.

[4] I. Viola, D. Pisignano, R. Cingolani, G. Gigli, Anal. Chem. 77 (2005) 591595.

[5] X.M. Zhao, Y. Xia, G.M. Whitesides, J. Mater. Chem. 7 (1997) 10691074 .

[6] D. Pisignano, E. Sariconi, M. Mazzeo, G. Gigli, R. Cingolani, Adv. Mater. 14 (2002) 1565-1567.

[7] A. Han, G. Mondin, N.G. Hegelbach, N.F. de Rooij, U. Staufer, J. Colloids Interef. Sci. 293 (2006) 151-158.

[8] A.A. Darhuber, S.M. Troian, Phys. Rev. E 64 (2001) 316.

[9] D. Myers, Surface, Interface and Colloids: Principle and Applications, Wiley-VCH, NY, 1999.

[10] E.W. Washburn, Phys. Rev. 17 (1921) 273.

[11] A. Groisman, V. Steinberg, Nature 405 (2000) 53; A. Groisman, V. Steinberg, Nature 410 (2001) 905.

[12] M.D. Graham, Phys. Fluids 15 (2003) 1702.

[13] $T_{0}$ is defined, according to the free volume model, as the freezing temperature (Vogel temperature) at which the free volume approaches zero and hence the viscosity diverges toward very high values. $\eta_{0}$ is proportional to the viscosity in the high temperature limit $(T \rightarrow \infty)$. $D$ is related to the fragility, thus being connected either with the cooperative dynamics in the liquid (kinetic fragility) or with the heat capacity in a glassy state (thermodynamic fragility).

[14] Z.Y. Cheng, J. Polym. Sci.: B: Polym. Phys. 37 (1999) 983. 